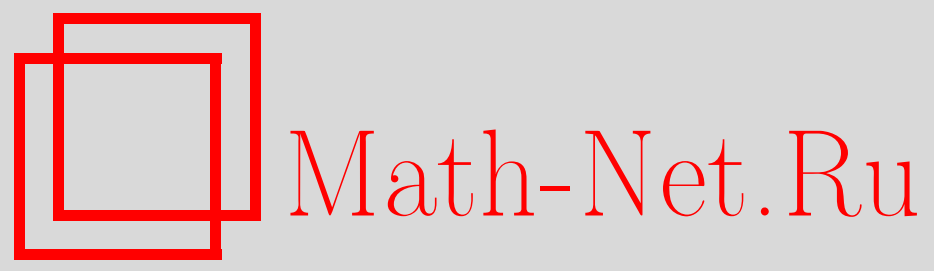

Д. К. Потапов, О характере разрывов нелинейности в задачах на собственные значения для уравнений эллиптического типа, Вестн. Сам. гос. техн. ун-та. Сер. Физ.-мат. науки, 2012, выпуск 3(), 188-190

DOI: https://doi.org/10.14498/vsgtu1053

Использование Общероссийского математического портала Math-Net.Ru подразумевает, что вы прочитали и согласны с пользовательским соглашением

http://www . mathnet.ru/rus/agreement

Параметры загрузки:

IP : 44.207 .124 .84

26 апреля 2023 г., 12:51:05

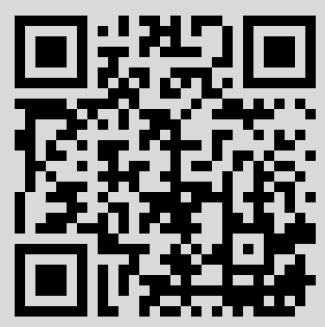




\title{
О ХАРАКТЕРЕ РАЗРЫВОВ НЕЛИНЕЙНОСТИ В ЗАДАЧАХ НА СОБСТВЕННЫЕ ЗНАЧЕНИЯ ДЛЯ УРАВНЕНИЙ ЭЛЛИПТИЧЕСКОГО ТИПА
}

\author{
Д. К. Потапов \\ Санкт-Петербургский государственный университет, \\ 198504, Россия, Санкт-Петербург, Университетский просп., 35. \\ E-mail: potapov@apmath.spbu.ru
}

\begin{abstract}
Рассматриваются задачи на собственные значения для уравнений эллиптического типа с разрывными по фазовой переменной нелинейностями. Исследуется характер разрывов нелинейности в таких задачах. В отличие от работ других авторов, в данной статъе ослаблены ограничения на точки разрыва нелинейности.
\end{abstract}

Ключевые слова: краевые задачи, уравнения эллиптического типа, задачи на собственные значения, разрывная нелинейность, характер разрывов.

В течение ряда лет автор изучает основные краевые задачи для уравнений эллиптического типа со спектральным параметром и разрывной по фазовой переменной нелинейностью (см., например, работы [1-7]). В работах других отечественных математиков в последние годы нелинейные задачи на собственные значения для уравнений эллиптического типа с разрывными нелинейностями не рассматривались. В работах зарубежных математиков последних лет (см., например, работы [8-14]) на разрывы нелинейности $g(x, u)$ по фазовой переменной $u$ накладывается следующее достаточно жёсткое ограничение - существует множество $\Omega_{0} \subset \Omega$ меры нуль, для которого объединение

$$
\bigcup_{x \in \Omega \backslash \Omega_{0}}\{u \in \mathbb{R}: g(x, \cdot) \text { разрывна в точке } u\}
$$

имеет меру нуль $(\Omega$ - область, в которой рассматривается краевая задача). По сравнению с работами [8-14] в работах автора ослаблены ограничения на точки разрыва нелинейности $g(x, u)$ по $u$, а именно на разрывы функции $g(x, \cdot)$ накладывается условие $g(x, u-)<g(x, u+)$, где $g(x, u \pm)=\lim _{s \rightarrow u \pm 0} g(x, s)$. Таким образом, в работах автора не предполагается, что проекция множества точек разрыва $g(x, u)$ по $u$ на ось фазовой переменной $u$ имеет меру нуль в $\mathbb{R}$, что достаточно существенно. Это важно и для ряда прикладных задач. Например, в задаче об отрывных течениях несжимаемой жидкости М. А. Гольдштика [15] отрывные течения формируются на множестве ненулевой меры, когда разрывы прыгающие $[16,17]$ (т. е. если $u$ - точка разрыва функции $g(x, \cdot)$, то $g(x, u-)<g(x, u+))$. Ограничение на множество точек разрыва нелинейности можно заменить на более общее - А-условие.

ОпРЕДЕлЕНИЕ. Для дифференциального уравнения с дифференциальным оператором $L$ выполнено $A$-условие, если найдётся не более чем счётное семейство поверхностей $\left\{S_{i}, i \in I\right\}, S_{i}=\left\{(x, u) \in \mathbb{R}^{n+1}: u=\varphi_{i}(x), x \in \Omega\right\}, \varphi_{i} \in W_{1, \text { loc }}^{2}(\Omega)$, для которых при почти всех $x \in \Omega$ неравенство $g(x, u-)>g(x, u+)$ влечёт существование $i \in I$ такого, что $u=\varphi_{i}(x)$ и $\left(L \varphi_{i}(x)-g\left(x, \varphi_{i}(x)-\right)\right)\left(L \varphi_{i}(x)-g\left(x, \varphi_{i}(x)+\right)\right)>0$.

Такое условие предполагалось, например, в работе [18]. Отметим, что А-условие запрещает при почти всех $x$ выход решения $u(x)$ краевой задачи на поверхности раз-

Дмитрий Константинович Потапов (к.ф.-м.н., доц.), доцент, каф. высшей математики. 
рывов нелинейности $g(x, u)$ в точках так называемых падающих разрывов по фазовой переменной, т. е. точках, для которых $g(x, u-)>g(x, u+)$. Таким образом, если для почти всех $x \in \Omega$ верно неравенство $g(x, u-)<g(x, u+) \forall u \in \mathbb{R}$, т. е. все разрывы по фазовой переменной $u$ - прыгающие, то А-условие для уравнения выполняется. Несложно привести достаточные и легко проверяемые признаки выполнимости Аусловия, выраженные в терминах коэффициентов дифференциального оператора и нелинейности, а также дать физическую трактовку этому условию.

\section{БИБЛИОГРАФИЧЕСКИЙ СПИСОК}

1. Павленко В. Н., Потапов Д. К. О существовании луча собственных значений для уравнений с разрывными операторами // Сиб. матем. журн., 2001. Т. 42, № 4. С. 911919; англ. пер.: Pavlenko V. N., Potapov D. K. Existence of a ray of eigenvalues for equations with discontinuous operators // Siberian Math. J., 2001. Vol.42, no. 4. Pp. 766773.

2. Павленко В. Н., Потапов Д. К. Аппроксимация краевых задач эллиптического типа со спектральным параметром и разрывной нелинейностью // Изв. вузов. Матем., 2005. № 4. C. 49-55; англ. пер.: Pavlenko V. N., Potapov D. K. Approximation of boundaryvalue problems of elliptic type with a spectral parameter and discontinuous nonlinearity // Russian Math. (Iz. VUZ), 2005. Vol. 49, no. 4. Pp. 46-52.

3. Потапов Д. К. Об одной оценке сверху величины бифуркационного параметра в задачах на собственные значения для уравнений эллиптического типа с разрывными нелинейностями // Дифферени. уравнения, 2008. Т. 44, № 5. С. 715-716; англ. пер.: Potapov D. K. On an upper bound for the value of the bifurcation parameter in eigenvalue problems for elliptic equations with discontinuous nonlinearities // Differ. Equ., 2008. Vol. 44, no. 5. Pp. 737-739.

4. Потапов Д. К. О структуре множества собственных значений для уравнений эллиптического типа высокого порядка с разрывными нелинейностями // Дифферени. уравнения, 2010. T. 46, № 1. С. 150-152; англ. пер.: Potapov D. K. On the eigenvalue set structure for higher-order equations of elliptic type with discontinuous nonlinearities // Differ. Equ., 2010. Vol. 46, no. 1. Pp. 155-157.

5. Потапов Д. К. Оценки дифференциального оператора в задачах со спектральным параметром для уравнений эллиптического типа с разрывными нелинейностями // Вестн. Сам. гос. техн. ун-та. Сер. Физ.-мат. науки, 2010. №5(21). С. 268-271. [Potapov D. K. Estimations of a differential operator in spectral parameter problems for elliptic equations with discontinuous nonlinearities // Vestn. Samar. Gos. Tekhn. Univ. Ser. Fiz.-Mat. Nauki, 2010. no. 5(21). Pp. 268-271].

6. Потапов Д. К. Бифуркационные задачи для уравнений эллиптического типа с разрывными нелинейностями // Матем. заметки, 2011. Т.90, №2. С. 280-284; англ. пер.: Potapov D. K. Bifurcation problems for equations of elliptic type with discontinuous nonlinearities // Math. Notes, 2011. Vol. 90, no. 2. Pp. 260-264.

7. Потапов Д. К. О количестве решений в задачах на собственные значения для уравнений эллиптического типа с разрывными нелинейностями // Becmн. Сам. гос. техн. ун-та. Сер. Физ.-мат. науки, 2012. №1(26). С. 251-255. [Potapov D. K. On number of solutions in eigenvalue problems for elliptic equations with discontinuous nonlinearities // Vestn. Samar. Gos. Tekhn. Univ. Ser. Fiz.-Mat. Nauki, 2012. no. 1(26). Pp. 251-255].

8. Marano $S$. A. Elliptic eigenvalue problems with highly discontinuous nonlinearities // Proc. Amer. Math. Soc., 1997. Vol. 125, no. 10. Pp. 2953-2961.

9. Marano S. A., Motreanu D. On a three critical points theorem for non-differentiable functions and applications to nonlinear boundary value problems // Nonlinear Anal., 2002. Vol. 48, no. 1. Pp. 37-52.

10. Bonanno G. Some remarks on a three critical points theorem// Nonlinear Anal., 2003. Vol. 54, no. 4. Pp. 651-665.

11. Bonanno G., Giovannelli N. An eigenvalue Dirichlet problem involving the $p$-Laplacian with discontinuous nonlinearities // J. Math. Anal. Appl., 2005. Vol. 308, no. 2. Pp. 596-604. 
12. Zhang G., Liu S. Three symmetric solutions for a class of elliptic involving the $p$-Laplacian with discontinuous nonlinearities in $\mathbb{R}^{n} / /$ Nonlinear Anal., 2007. Vol.67, no. 7. Pp. 22322239 .

13. Bonanno G., Candito $P$. Non-differentiable functionals and applications to elliptic problems with discontinuous nonlinearities // J. Differential Eq., 2008. Vol. 244, no. 12. Pp. 3031-3059.

14. Bonanno G., Chinni A. Discontinuous elliptic problems involving the $p(x)$-Laplacian // Math. Nachr., 2011. Vol. 284, no. 5-6. Pp. 639-652.

15. Голъдитик M. А. Математическая модель отрывных течений несжимаемой жидкости // Докл. АН СССР, 1962. Т. 147, №6. С. 1310-1313; англ. пер.: Gol'dshtik M. A. А mathematical model of separated flows in an incompressible liquid // Soviet. Math. Dokl., 1963. Vol. 7. Pp. 1090-1093.

16. Потапов Д. К. Математическая модель отрывных течений несжимаемой жидкости // Изв. РАЕН. Сер. МММИУ, 2004. Т. 8, № 3-4. С. 163-170. [Potapov D. K. A mathematical model of separated flows in an incompressible fluid // Izv. RAEN. Ser. MMMIU, 2004. Vol. 8, no. 3-4. Pp. 163-170].

17. Потапов Д. К. Непрерывные аппроксимации задачи Гольдштика // Maтем. заметки, 2010. T. 87, № 2. C. 262-266; англ. пер.: Potapov D. K. Continuous approximations of Gol'dshtik's model // Math. Notes, 2010. Vol. 87, no. 2. Pp. 244-247.

18. Потапов Д. К. Аппроксимация задачи Дирихле для уравнения эллиптического типа высокого порядка со спектральным параметром и разрывной нелинейностью // Дифферени. уравнения, 2007. Т. 43, № 7. С. 1002-1003; англ. пер.: Potapov D. K. Approximation to the Dirichlet problem for a higher-order elliptic equations with a spectral parameter and a discontinuous nonlinearity // Differ. Equ., 2007. Vol.43, no. 7. Pp. 1031-1032.

Поступила в редакцию 01/IV/2012;

в окончательном варианте - 10/VIII/2012.

MSC: 35P30; $35 \mathrm{~J} 60$

\section{ON THE CHARACTER OF NONLINEARITY DISCONTINUITIES IN EIGENVALUE PROBLEMS FOR ELLIPTIC EQUATIONS}

\section{K. Potapov}

St. Petersburg State University,

35, Universitetskiy prosp., St. Petersburg, 198504, Russia.

E-mail: potapov@apmath.spbu.ru

The eigenvalue problems for equations of elliptic type with discontinuous by the phase variable nonlinearities are considered. The character of nonlinearity discontinuities is investigated. In this paper the restrictions on discontinuity points of nonlinearity are weaker than in works of other authors.

Key words: boundary value problems, elliptic equations, eigenvalue problems, discontinuous nonlinearity, character of discontinuities.

Original article submitted 01/IV/2012;

revision submitted 10/VIII/2012.

Dmitriy K. Potapov (Ph. D. (Phys. \& Math.)), Associate Professor, Dept. of Higher Mathematics. 